\title{
Oscillator Strengths for Fe II Transitions at 224.918 and 226.008 Nanometers
}

\author{
Scott D. Bergeson \\ scott.bergeson@byu.edu \\ K. L. Mullman \\ J. E. Lawler
}

Follow this and additional works at: https://scholarsarchive.byu.edu/facpub

Part of the Astrophysics and Astronomy Commons

\section{Original Publication Citation}

S. D. Bergeson, K. L. Mullman, and J. E. Lawler. Oscillator strengths for Fe II transitions at 224.918 and 226.008 nanometers. Astrophys. J. 435 (2), L157-L159 (1994).

\section{BYU ScholarsArchive Citation}

Bergeson, Scott D.; Mullman, K. L.; and Lawler, J. E., "Oscillator Strengths for Fe II Transitions at 224.918 and 226.008 Nanometers" (1994). Faculty Publications. 1827.

https://scholarsarchive.byu.edu/facpub/1827 


\title{
OSCILLATOR STRENGTHS FOR Fe II TRANSITIONS AT 224.918 AND 226.008 NANOMETERS
}

\author{
S. D. Bergeson, K. L. Mullman, And J. E. Lawler \\ Physics Department, U.W.-Madison, Madison, WI 53706 \\ Received 1994 July 22; accepted 1994 August 24
}

\begin{abstract}
We report accurate experimental absorption oscillator strengths ( $f$-values) for transitions out of the ground level of $\mathrm{Fe}$ II to the $z^{4} D_{7 / 2}^{o}$ and $z^{4} F_{9 / 2}^{o}$ levels at 224.918 and $226.008 \mathrm{~nm}$ (air wavelengths) to be $0.00182(14)$ and $0.00244(19)$, respectively. The number in parenthesis is the uncertainty in the last digits. These two lines are important for studying Fe abundances and grain depletions in the interstellar medium. These $f$-values are determined by combining emission branching fractions with radiative lifetimes. Branching fractions are measured using classical spectroradiometry on an optically thin source. Radiative lifetimes are from the literature.
\end{abstract}

Subject headings: atomic data - ISM : abundances - ISM : atoms - ultraviolet

\section{INTRODUCTION}

Gaseous iron in the diffuse, low-temperature interstellar medium (ISM) is predominantly singly ionized and in the ground electronic level. Accurate spectroscopic abundance determinations of iron in the gas phase of the ISM can be made using ultraviolet transitions from the ground level provided a reliable $f$-value is known. Absorption features from gaseous iron in the ISM of intervening galaxies are seen in absorption line spectra of quasars. Because the abundance of iron is high, a weak transition is required to keep absorption measurements on or near the linear part of the curve of growth. The weakest absorption lines with published detections are 224.918 and $226.008 \mathrm{~nm}$. These lines have been identified as key lines for understanding $\mathrm{Fe}$ abundances in the ISM (Savage 1993; Shull 1993; York 1993). Published $f$-values for these transitions vary by almost a factor of 2 (Martin, Fuhr, \& Wiese 1988; Kurucz 1988; Fawcett 1988; Shull, Van Steenberg, \& Seab 1983; Van Buren 1986). We report new accurate experimental $f$-values for these transitions. The $f$-values are determined by combining precise radiative lifetimes from the literature with our emission branching fraction measurements. The emission branching fraction measurements are made using classical spectroradiometry on an optically thin hollow cathode discharge using a 3 meter focal length vacuum echelle spectrometer. This method has been used to provide accurate $f$-value determinations for the Si II $180.8 \mathrm{~nm}$ transition (Bergeson \& Lawler 1993a), the $\mathrm{Si}$ II intersystem transitions (Calamai, Smith, \& Bergeson 1993), and the $\mathrm{Zn}$ II and $\mathrm{Cr}$ II resonance transitions (Bergeson \& Lawler 1993b).

\section{BRANCHING FRACTIONS}

The branching fraction measurements are made using classical spectroradiometry on an optically thin hollow cathode discharge. The apparatus consists mainly of three items: the iron hollow cathode discharge, the high resolution spectrometer, and the photomultiplier (PMT) detector/data recording system.

The iron hollow cathode discharge is of our own design. It is a water-cooled, open ended discharge. The cathode is $1 \mathrm{~cm}$ inner diameter, $10 \mathrm{~cm}$ long. Discharge currents from $20 \mathrm{~mA}$ to $1 \mathrm{~A}$ are used in this experiment. The discharge is run with either $\mathrm{Ne}$ or Ar as a buffer gas. Varying the buffer gas allows us to check for $\mathrm{Ne}$ and Ar blends with Fe II emission lines. Emis- sion from Fe II levels is optimized at 12 mtorr $\mathrm{Ar}$ or $30 \mathrm{mtorr}$ $\mathrm{Ne}$.

The high resolution spectrometer is a McPherson 2173 vacuum echelle spectrometer. The observed resolving power of this 3 meter focal length instrument exceeds 600,000 . A high resolving power is required for sorting out a complex spectrum such as Fe II. Because the instrument is operated in high order, 22-26 for this experiment, a pre-monochromator (McPherson model 218) is used as an order sorter. The spectral limit of resolution of the 3 meter echelle is smaller than the apparent line width from the hollow cathode discharge $(\lambda / \Delta \lambda \approx 200,000)$.

The PMT is a Hamamatsu R1220 solar blind tube. It is verified to be linear over the signal levels used in this experiment. The PMT is used in "analog" mode, meaning that the photo-current is measured as a function of time as the grating is rotated. High signal-to-noise $(\mathrm{S} / \mathrm{N}>50)$ is common because the tube is relatively quiet. In coming months this spectrometer will be equipped with a deep ultraviolet sensitive CCD detector array, vastly improving the data handling capability of this project. We are planning a much larger set of $\mathrm{Fe}$ II $f$-value measurements in collaboration with Sveneric Johansson at Lund University in Sweden. These initial measurements are being published separately because of the high level of interest in the weak $\mathrm{Fe}$ II resonance lines.

The Fe II transitions are identified by comparing the emission from the hollow cathode discharge with published line lists (Dobbie 1938; Crosswhite 1975; Johansson 1978). A sample emission spectrum covering the $224.918 \mathrm{~nm}$ line is shown in Figure 1. The high resolving power and high signalto-noise ratio are apparent. The line at $224.920 \mathrm{~nm}$ is identified by Johansson (1978) as connecting the $e^{6} F_{3 / 2}$ and $z^{6} D_{3 / 2}^{o}$ levels.

The emission source is largely stable over time, but small variations in the emission signal are seen. We account for these small changes by alternately measuring different lines from the same upper level, and averaging the results. This ensures that any fluctuations in the emission from the hollow cathode discharge over time are averaged out. The apparent branching fractions are measured while varying the slit width of the spectrometer by a factor of 10 , to check for blends or other line structure, and while varying the current by a factor of 50 , to check for radiation trapping. We find a low current region where the apparent branching ratio for strong lines is independent of current.

A complete set of branching ratio measurements (or branch- 


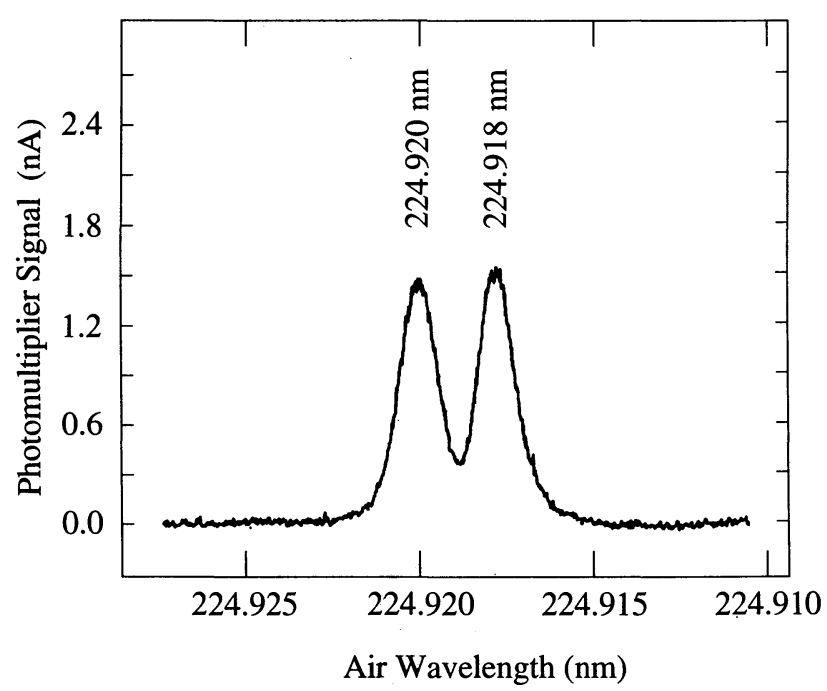

Fig. 1.-Sample emission spectrum, for $\mathrm{Fe}$ II $224.918 \mathrm{~nm}$ connecting the $a^{6} D_{9 / 2}$ ground level to the $z^{4} D_{7 / 2}^{o}$ level. The high resolution and high signal-tonoise ratio are apparent. The Fe II line at $224.920 \mathrm{~nm}$ connects the $e^{6} F_{3 / 2}$ level to the $z^{6} D_{3 / 2}^{o}$ level.

ing fractions) is required to accurately determine transition probabilities by normalizing to radiative lifetimes. Completeness of the transitions for these levels is judged by identifying all decay branches obeying parity change and $|\Delta J| \leq 1$ selection rules using published energy levels (Sugar \& Corliss 1985). The list of all possible allowed transitions is compared to lists of observed transitions (Dobbie 1938; Crosswhite 1975; Johansson 1978). We measure all observed branches from the $3 d^{6}\left({ }^{5} D\right) 4 p z^{4} D_{7 / 2}^{o}$ and the $3 d^{6}\left({ }^{5} D\right) 4 p z^{4} F_{9 / 2}^{o}$ levels with wavelengths less than $300 \mathrm{~nm}$. For longer wavelengths, we consider branching fraction measurements and calculations from the NBS critical compilation of $f$-values (Fuhr, Martin, \& Wiese 1988), Fawcett (1988), and Kurucz (1988). These three studies find that wavelengths above $300 \mathrm{~nm}$ contribute $3.27(56) \%$ and $0.54(42) \%$ of the total decay from the $3 d^{6}\left({ }^{5} D\right) 4 p z^{4} D_{7 / 2}^{o}$ and $3 d^{6}\left({ }^{5} D\right) 4 p z^{4} F_{9 / 2}^{o}$ levels, respectively. The uncertainty in these numbers is the $1 \sigma$ statistical variation in the reported numbers. The sum of a complete set of branching fractions equals 1.0 by definition. In our experiment, the sum of our branching fractions for transitions less than $300 \mathrm{~nm}$ is set to 0.9673 for the $3 d^{6}\left({ }^{5} D\right) 4 p z^{4} D_{7 / 2}^{o}$ level, and the sum of our branching fractions for transitions less than $300 \mathrm{~nm}$ is set to 0.9946 for the $3 d^{6}\left({ }^{5} D\right) 4 p z^{4} F_{9 / 2}^{o}$ level. The uncertainties in these corrections to our measured branching fractions at wavelengths less than $300 \mathrm{~nm}$ are not serious because the corrections are small.

The relative response of the spectrometer-PMT detection system is calibrated as a function of wavelength using a NIST traceable Optronics $\mathrm{D}_{2}$ lamp and verified using a NIST calibrated argon mini-arc (Bridges \& Ott 1977). The Optronics $D_{2}$ lamp is a secondary standard of spectral irradiance, and should not be imaged onto the spectrometer entrance slit. This means that only parts of the spectrometer grating and mirrors are illuminated in calibration run. We compensate for this by varying the position of the $\mathrm{D}_{2}$ lamp in a plane perpendicular to the optical axis of the system and find no variations in the performance of the system. Scattered light in the spectrometer presents a potential systematic error for the efficiency calibration. The scattered light level is determined by measuring the photon flux between orders of the 3 meter spectrometer.
The scattered light level correction is typically only a few percent, and can be subtracted with confidence.

We measure a few branching ratios for well isolated transitions using an Acton Research Corporation 1 meter focal length vacuum compatible spectrometer. This is a lower resolution instrument $(\lambda / \Delta \lambda \approx 60,000)$, with a larger etendue. We are able to measure ratios of the strongest lines for each level at extremely low discharge currents $(20 \mathrm{~mA})$ and good signal-to-noise. We obtain branching ratios agreeing with our data from the 3 meter instrument at the level of a few percent. This gives us confidence that the transitions involved are optically thin, and that the optical calibration of the 3 meter high resolution spectrometer is correct. Using the 1 meter spectrometer, we compare the NIST-calibrated argon mini-arc and the Optronics $D_{2}$ lamp. Special care is taken to eliminate systematic errors which could arise while comparing a secondary standard of spectral radiance (the NIST argon mini-arc) and a secondary standard of spectral irradiance (the Optronics $\mathrm{D}_{2}$ lamp). For the wavelength range covered in this study, the relative wavelength calibration of the two lamps agrees to well within the stated uncertainties.

The radiative lifetimes of both the $z^{4} D_{7 / 2}^{o}$ and the $z^{4} F_{9 / 2}^{o}$ levels are well known. An early laser induced fluorescence measurement on the $z^{4} D_{7 / 2}^{o}$ level by Hannaford \& Lowe (1983) was somewhat less accurate than claimed. This was likely due to electronic bandwidth limitations in the time resolved fluorescence detection system. Salih \& Lawler (1983) measured the lifetime of the $z^{4} F_{9 / 2}^{o}$ level using time resolved laser induced fluorescence and reported 3.9(2) ns. This number was confirmed and refined by Guo et al. (1992) who reported lifetimes of 3.87(9) nm for the $z^{4} F_{9 / 2}^{o}$ level and 3.02(6) ns for the $z^{4} D_{7 / 2}^{o}$ level. Although the fast beam-laser method used by Guo et al. is more difficult and time consuming than the time resolved laser induced fluorescence method, it is believed to be extremely accurate for these short-lived levels. Hannaford et al. (1992) using time resolved laser-induced fluorescence have also confirmed the lifetime measurements by Guo et al. We use the lifetime measurements by Guo et al. (1992) to normalize our branching fractions and include $2 \sigma$ uncertainties from the lifetimes in our final transition probabilities.

\section{RESULTS}

The branching fractions and transition probabilities of all transitions out of the $3 d^{6}\left({ }^{5} D\right) 4 p z^{4} D_{7 / 2}^{o}$ and the $3 d^{6}\left({ }^{5} D\right) 4 p z^{4} F_{9 / 2}^{o}$ levels are listed in Table 1 . The level designations are from Sugar $\&$ Corliss (1985). Branching fractions and transition probabilities measured in this experiment are compared to others in the literature. In particular, Table 1 contains data from workers who measured or calculated complete sets of transition probabilities out of the $3 d^{6}\left({ }^{5} D\right) 4 p z^{4} D_{7 / 2}^{o}$ and $3 d^{6}\left({ }^{5} D\right) 4 p z^{4} F_{9 / 2}^{o}$ levels. The number in parenthesis beside each value is the uncertainty in the last digit(s). The uncertainties in our branching fraction measurements in most cases are dominantly systematic uncertainties stemming from our radiometric calibration. The transition probabilities from the NBS critical compilation (Fuhr, Martin, \& Wiese 1988) are actually from branching fractions normalized to radiative lifetimes by Hannaford \& Lowe (1983). The branching fractions were measured using the Fourier Transform Spectrometer at Kitt Peak, with supplemental measurements made on a grating spectrometer. The transition probabilities from Kurucz (1988) and from Fawcett (1988) are theoretical. Kurucz's transition probabilities best compare with our results for the strongest lines. His values for the lines at 273.955 and $275.573 \mathrm{~nm}$ agree 
TABLE 1

Branching Fractions and Absolute Transition Probabilities for Lines from the $z^{4} D_{7 / 2}^{o}$ AND $z^{4} F_{9 / 2}^{o}$ Levels in Fe II

\begin{tabular}{|c|c|c|c|c|c|c|c|c|c|c|}
\hline \multirow[b]{2}{*}{$\begin{array}{l}\text { UPPER } \\
\text { LEVEL }\end{array}$} & \multirow[b]{2}{*}{$\begin{array}{c}\text { LOWER } \\
\text { LEVEL } \\
\end{array}$} & \multirow[b]{2}{*}{$\lambda_{\text {air }}(\mathrm{nm})$} & \multicolumn{4}{|c|}{ BRANCHING FRACTIONS } & \multicolumn{4}{|c|}{ Transition Probabilities $\left(10^{8} \mathrm{~s}^{-1}\right)$} \\
\hline & & & This Work & $\mathrm{NBS}^{\mathrm{a}}$ & $F^{\mathbf{b}}$ & $\mathrm{Ku}^{\mathrm{c}}$ & $\begin{array}{l}\text { This } \\
\text { Work }\end{array}$ & $\mathrm{NBS}^{\mathrm{a}}$ & $\mathrm{F}^{\mathrm{b}}$ & $\mathrm{Ku}^{\mathrm{c}}$ \\
\hline \multirow[t]{8}{*}{$z^{4} D_{7 / 2}^{o} \ldots \ldots$} & $\begin{array}{l}a^{6} D_{9 / 2} \\
a^{6} D_{7 / 2}\end{array}$ & $\begin{array}{l}224.918 \\
226.882\end{array}$ & $\begin{array}{l}0.91(6) \% \\
0.120(12)\end{array}$ & $1.5 \%$ & $0.66 \%$ & $\begin{array}{l}1.2 \% \\
0.21\end{array}$ & $\begin{array}{l}0.0300(23) \\
0.0040(4)\end{array}$ & $0.041(10)$ & 0.028 & $\begin{array}{l}0.050 \\
0.0086\end{array}$ \\
\hline & $a^{4} F_{9 / 2}$ & 234.811 & $19.5(10)$ & 19.0 & 23.4 & 29.1 & $0.65(5)$ & $0.51(8)$ & 1.00 & 1.20 \\
\hline & $a^{4} F_{7 / 2}$ & 237.927 & $7.33(15)$ & 5.6 & 6.8 & 7.3 & $0.243(12)$ & $0.15(2)$ & 0.29 & 0.30 \\
\hline & $a^{4} F_{5 / 2}$ & 240.260 & $0.65(6)$ & 0.71 & & 0.58 & $0.0216(22)$ & $0.019(10)$ & & 0.024 \\
\hline & $a^{4} D_{7 / 2}$ & 273.955 & $68.2(11)$ & 70.7 & 66.3 & 57.7 & $2.26(11)$ & $1.90(19)$ & 2.83 & 2.38 \\
\hline & $a^{4} P_{5 / 2}$ & 322.773 & $\ldots$ & 2.2 & 2.6 & 2.7 & $\ldots$ & $0.059(6)$ & 0.11 & 0.11 \\
\hline & $b^{4} P_{5 / 2}^{3 / 2}$ & 423.317 & $\ldots$ & 0.17 & & 0.16 & $\ldots$ & $0.0047(12)$ & & 0.0068 \\
\hline & $b^{4} F_{9 / 2}^{j / 2}$ & 458.383 & $\ldots$ & 0.14 & 0.10 & 0.15 & $\ldots$ & $0.0038(19)$ & 0.0044 & 0.0063 \\
\hline \multirow[t]{7}{*}{$z^{4} F_{9 / 2}^{o} \ldots \ldots$} & $a^{6} D_{9 / 2}$ & 226.008 & $1.24(8)$ & 2.0 & 0.98 & 1.2 & $0.0319(25)$ & $0.049(25)$ & 0.033 & 0.036 \\
\hline & $a^{6} D_{7 / 2}^{9 / 2}$ & 227.992 & $1.74(4)$ & 1.6 & 1.4 & 1.8 & $0.0449(23)$ & $0.039(10)$ & 0.046 & 0.055 \\
\hline & $a^{4} F_{9 / 2}$ & 236.000 & $12.7(7)$ & 9.8 & 13.0 & 17.2 & $0.329(24)$ & $0.240(60)$ & 0.43 & 0.51 \\
\hline & $a^{4} F_{7 / 2}^{5 / 2}$ & 239.148 & $1.42(9)$ & 1.1 & 1.3 & 1.6 & $0.0366(30)$ & $0.027(13)$ & 0.042 & 0.047 \\
\hline & $a^{4} D_{7 / 2}$ & 275.573 & $82.4(8)$ & 85.4 & 83 & 78.0 & $2.13(10)$ & $2.10(21)$ & 2.77 & 2.34 \\
\hline & $b^{4} F_{9 / 2}$ & 462.934 & $\ldots$ & 0.053 & & 0.051 & $\ldots$ & $0.0013(7)$ & & 0.0015 \\
\hline & $a^{4} G_{11 / 2}$ & 531.662 & & 0.13 & 0.092 & 0.093 & $\cdots$ & $0.0033(8)$ & 0.0031 & 0.0028 \\
\hline
\end{tabular}

${ }^{a}$ NBS critical compilation (Fuhr et al. 1988).

${ }^{b}$ Fawcett 1988.

c Kurucz 1988.

TABLE 2

ABSORPTION $f$-VALUES FOR 224.918 AND 226.008 NANOMETER TRANSITIONS IN Fe II

\begin{tabular}{|c|c|c|}
\hline Study & $224.918 \mathrm{~nm}$ & $226.008 \mathrm{~nm}$ \\
\hline This Work & 0.00182 & 0.002 \\
\hline NBS (Fuhr et al. 1988) & $0.0025(6)$ & $0.0037(19)$ \\
\hline Kurucz 1988 . & 0.00303 & 0.00279 \\
\hline Fawcett 1988. & 0.0017 & 0.0025 \\
\hline Shull et al. 1983 & & $0.0028(14)$ \\
\hline de Boer et al. 1987 & 0.0018 & \\
\hline Van Buren 1986 & & $0.0046(16)$ \\
\hline
\end{tabular}

with our values to $5.3 \%$ and $9.9 \%$, respectively. However, the average percent difference between Kurucz's values and our results for all lines is $+51.0 \%$ and $+25.7 \%$ for the $z^{4} D_{7 / 2}^{o}$ and $z^{4} F_{9 / 2}^{o}$ levels, respectively. Fawcett's transition probabilities agree best with ours for the weakest lines: $-6.7 \%$ and $+3.4 \%$ for the 224.918 and $226.008 \mathrm{~nm}$ lines. The average percent difference between Fawcett's values and our results for all lines is $+22.9 \%$ and $+16.3 \%$ for the $z^{4} D_{7 / 2}^{o}$ and $z^{4} F_{9 / 2}^{o}$ levels.

In Table 2, our transition probabilities for the 224.918 and $226.008 \mathrm{~nm}$ transitions are converted to $f$-values and compared to other values in the literature. Van Buren (1986), Shull et al. (1983), and de Boer et al. (1987) have deduced $f$-values from a curve of growth analysis of Fe II observations of the ISM. The latter two analyses used experimental $f$-values from Assousa \& Smith (1972) to normalize $f$-values from the ISM observations. The results agree quite well with ours. The $f$-value for the $224.918 \mathrm{~nm}$ transition from de Boer, Jura, \& Shull (1987) agrees with our value to approximately $1 \%$. The $f$-value for the $226.008 \mathrm{~nm}$ transition from Shull et al. (1983) [which is the same as the theoretical determination from Kurucz (1988)] agrees with our value to $15 \%$. Morton (1991) critically reviewed $f$-values of resonance lines longward of $\mathrm{Ly} \alpha$ prior to 1991. Morton adopted the values published in the NBS critical compilation (Fuhr et al. 1988).

\section{CONCLUSION}

The $f$-values for the $224.918 \mathrm{~nm}[f=0.00182(14)]$ and $226.008 \mathrm{~nm}[f=0.00244(19)]$ transitions from the ground level to the $3 d^{6}\left({ }^{5} D\right) 4 p z^{4} D_{7 / 2}^{o}$ and the $3 d^{6}\left({ }^{5} D\right) 4 p z^{4} F_{9 / 2}^{o}$ levels are determined by combining radiative lifetimes and emission branching fractions. These measurements will put abundance measurements of gaseous iron in the ISM on a firmer, more quantitative footing. The planned larger set of Fe in $f$-value measurements described earlier will include the important $\mathrm{Fe}$ II line at $\lambda_{\text {vac }}=160.845 \mathrm{~nm}$.

This research supported by NASA under grant NAGW2908.

\section{REFERENCES}

Assousa, G., \& Smith, W. H. 1972, ApJ, 176, 259

Bergeson, S. D., \& Lawler, J. E. 1993a, ApJ, 414, L137

.1993b, ApJ, 408, 382

Bridges, J. M., \& Ott, W. R. 1977, Appl. Opt., 16, 367

Calamai, A. G., Smith, P. L., \& Bergeson, S. D. 1993, ApJ, 415, L59

Crosswhite, H. M. 1975, J. Res. NBS, 79A, 17

de Boer, K. S., Jura, M. A., \& Shull, J. M. 1987, in Exploring the Universe with the IUE Satellite, ed. Y. Kondo (Reidel: Dordrecht), 485

Dobbie, J. C. 1938, Ann. Solar Phys. Obs. Cambridge, 5, part 1, 1

Fawcett, B. C. 1988, Atomic Data Nucl. Data, 40, 1

Fuhr, J. R., Martin, G. A., \& Wiese, W. L. 1988, J. Phys. Chem. Ref. Data, 17, Suppl. 4, 108

Guo, B., Ansbacher, W., Pinnington, E. H., Ji, Q., \& Berends, R. W. 1992, Phys. Rev., A46, 641
Hannaford, P., \& Lowe, R. M. 1983, J. Phys. B, 16, L43

Hannaford, P., Lowe, R. M., Grevesse, N., \& Noels, A. 1992, ApJ, 259, 301

Johansson, S. 1978, Phys. Scripta, 18, 217

Kurucz, R. L. 1988, Trans. IAU, 28, 168

Morton, D. C. 1991, ApJS, 77, 119

Salih, S., \& Lawler, J. E. 1983, Phys. Rev., A28, 2653

Savage, B. D. 1993, Phys. Scripta, T47, 171

Shull, J. M. 1993, Phys. Scripta, T47, 165

Shull, J. M., Van Steenberg, M., \& Seab, C. G. 1983, ApJ, 271, 408

Sugar, J., \& Corliss, C. 1985, J. Phys. Chem. Ref. Data, 14, Suppl. 2, 420

Van Buren, D. 1986, ApJ, 311, 400

York, D. G. 1993, Phys. Scripta, T47, 176 